\title{
OPEN Gyroidal graphene/porous silicon array for exciting optical Tamm state as optical sensor
}

\author{
Zaky A. Zaky ${ }^{\circledR}$ \& Arafa H. Aly \\ In this study, the optical Tamm state is excited for the first time using gyroidal graphene/porous silicon \\ one-dimensional photonic crystal terminated by a gyroidal graphene layer. The gyroidal graphene and \\ porous silicon are used to enhance the figure of merit and sensitivity of the based Tamm resonance \\ photonic crystal sensor. By tuning different parameters like the angle of incidence, the thickness of \\ the sample layer, and the thickness of the gyroidal graphene layer, we have reached the optimized \\ sensor. The observation of resonant dips in the reflectance spectra is strong evidence that Tamm \\ plasmon-polaritons exist with higher sensitivity $(188.8 \mathrm{THz} / \mathrm{RIU})$ and figure of merit $\left(355,384 \mathrm{RIU}^{-1}\right)$ \\ than previously reported structures. The proposed sensor recorded sensitivity and FoM higher $38 \%$ \\ and $747 \%$ respectively than a similar structure composed of graphene sheets and porous silicon.
}

Tamm plasmon-polariton (TPP) is an optical resonance excited at the interface between metal and a periodic one-dimensional photonic crystal (1D-PC) ${ }^{1-9}$. Contrary to the conventional surface plasmon resonance, TPP can be excited for both electromagnetic polarizations, at any incident angle, and without using grating or a coupling prism ${ }^{10}$. Besides the fact that the incident electric field is localized within the defected $1 \mathrm{D}$-PC of the structure ${ }^{11-15}$, porous layers filled with analyte samples will be proposed in this study ${ }^{3,16}$. Recently, we proposed the excitation of TPP by porous silicon PC (PSi-PC) using graphene sheets ${ }^{17}$ or silver ${ }^{16}$. Porous silicon multilayers can be experimentally prepared by electrochemical etching with hydrogen fluoride as an electrolyte that is considered a simple one-step fabrication ${ }^{18,19}$.

In 2017, Bikbaev et al. investigated a TPP excited by metallic gyroid layer ${ }^{20}$. In 2020, Sun et al. fabricated a gyroid structure of bioinspired $\mathrm{Au}-\mathrm{CuS}$ to excite $\mathrm{SPR}^{21}$. In these calculations, we will use gyroidal graphene (GGr)/porous silicon array for the first time to excite TPP. The gyroidal structure possesses a surface with fixed mean curvature within a volumetric fill fractions range ${ }^{22}$. Gyroidal structures can be experimentally realized using inorganic templates from the butterfly nanostructure or self-assembly ${ }^{23}$, chemical vapor deposition ${ }^{24}$, self-assembly of a triblock copolymer ${ }^{25}$, selective laser melting ${ }^{26}$, solvent-free method $^{27}$, light-based $3 \mathrm{D}$ printing process $^{28}$, and controlled phase separation ${ }^{29}$. Graphene has very distinguished optical, electrical, and mechanical properties $^{6,30}$. Chemical doping or the use of an external gate voltage can change the carrier concentration of graphene layers $^{31}$. The negative conductivity of graphene at a certain frequencies makes it behave as a metalic layer ${ }^{32-36}$. So, the gyroidal graphene layer will be used to excite Tamm states.

\section{Materials and theoretical method}

The proposed structure will be $\left(\mathrm{GGr}_{1} / \mathrm{PSi}\right)^{\mathrm{N}} /$ cavity/GGr$/$ substrate as clear in Fig. $1 \mathrm{~A}$. N represents the number of PC periods. The optical permittivity of the graphene gyroid layer (Fig. 1B) will be calculated according to the following $\operatorname{model}^{20,38}$ :

$$
\varepsilon_{g y r}(\omega)=\frac{1_{g} \sqrt{2}}{a}\left[1-\left(\frac{4 \mathrm{r}_{g}}{\lambda_{g}}\right)^{2}\left(\frac{\pi \sqrt{-\varepsilon_{m}(\omega)}}{2 \sqrt{2} n}-1\right)^{2}\right],
$$

where a, $r_{g} f, \lambda_{g}, l_{g}$ are the gyroidal unit cell size (Fig. 1C), helix radius, the volume fraction of graphene in the gyroidal layer, effective plasmon frequency, and the wire turn length. The length of the wire ring (Fig. 1D) is calculated as: 

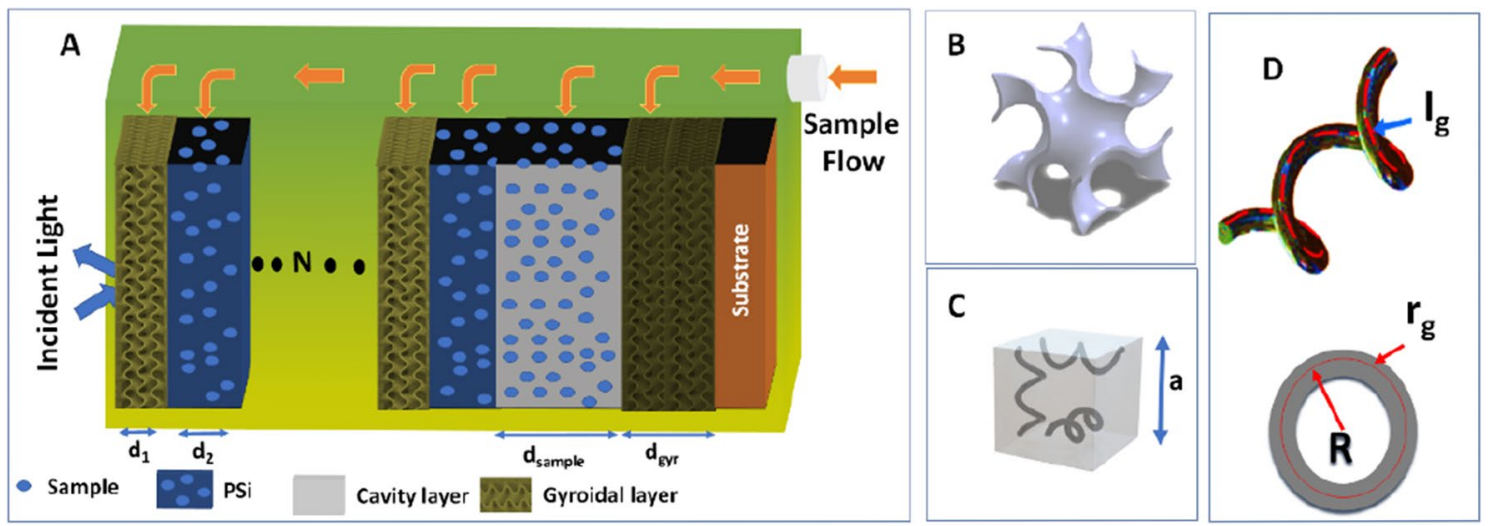

Figure 1. (A) The schematic structure of the gyroidal graphene PSi-PC, (B) gyroidal model ${ }^{37},(\mathbf{C})$ unit cell of tri-helical gyroid ${ }^{20}$, and (D) helix geometrical sizes ${ }^{20}$. For (B-D) (reprinted/adapted) with permission from ref. ${ }^{37}$ and ${ }^{20}$ respectively.

$$
l_{g}=\sqrt{(2 \pi R)^{2}+a^{2}}=a \sqrt{\frac{\pi^{2}(\sqrt{2}-1)^{2}}{4}+1},
$$

$\mathrm{R}, \mathrm{r}_{g}$ and $\lambda_{g}$ can be calculated as:

$$
\begin{gathered}
\mathrm{R}=\frac{(\sqrt{2}-1)^{2}}{4}+1, \\
\mathrm{r}_{g}=\mathrm{a} \sqrt{\mathrm{f}} \frac{\sqrt[4]{2}}{\sqrt{\pi\left[\sqrt{2+\pi^{2}}+\sqrt{\left.2+(3+2 \sqrt{2}) \pi^{2}\right]}\right.}}, \\
\lambda_{g}=1.15 \mathrm{a} \sqrt{1-0.65 \ln f} .
\end{gathered}
$$

According to the approximate local random phase, the conductivity of monolayers of graphene $(\sigma)$ in the $\mathrm{THz}$ frequency range is calculated as ${ }^{17,39,40}$ :

$$
\sigma=\mathrm{G} \frac{i e^{2} \mathrm{E}_{\mathrm{F}}}{\pi \hbar^{2}\left(\omega+\frac{i}{\tau}\right)},
$$

where $\mathrm{G}, \mathrm{E}_{\mathrm{F}}, \mathrm{e}, \omega, \tau$ are the graphene sheets' number, Fermi energy, the charge of the electron, angular frequency, and the relaxation time of the graphene sheet. Bruggeman's effective medium equation is used to calculate the optical refractive index of the PSi layer $\left(\mathrm{n}_{\mathrm{PSi}}\right)$ filled with sample $\left(\mathrm{n}_{\text {sample }}\right)$ with porosity $(\mathrm{P})^{41,42}$ :

$$
\begin{gathered}
\mathrm{n}_{\text {Psi }}=0.5 \sqrt{\psi+\sqrt{\psi^{2}+8 \mathrm{n}_{\text {si }}^{2} \mathrm{n}_{\text {sample }}^{2}}}, \\
\psi=3 \mathrm{P}\left(\mathrm{n}_{\text {sample }}^{2}-\mathrm{n}_{\mathrm{si}}^{2}\right)+\left(2 \mathrm{n}_{\mathrm{si}}^{2}-\mathrm{n}_{\text {sample }}^{2}\right) .
\end{gathered}
$$

The refractive index of silicon $\left(n_{S i}\right)$ in THz range is $3.42^{43}$. The transfer matrix method (TMM) is used to study the reflectance spectra of electromagnetic waves (TM mode) from the proposed structure as the following ${ }^{44-47}$ :

$$
\left(\begin{array}{c}
E_{0} \\
H_{0}
\end{array}\right)=\prod_{k=1}^{n} a_{k}\left(\begin{array}{c}
E_{n} \\
H_{n}
\end{array}\right)=\left[\begin{array}{ll}
A_{11} & A_{12} \\
A_{21} & A_{22}
\end{array}\right]\left(\begin{array}{c}
E_{n} \\
H_{n}
\end{array}\right)=A\left(\begin{array}{c}
E_{n} \\
H_{n}
\end{array}\right) .
$$

The $H_{n}$ and $E_{n}$ are the total magnetic and electric fields at the end of the proposed structure with $\mathrm{k}$ number of layers, $H_{0}$ and $E_{0}$ are total magnetic and electric fields at the air, and $a_{k}$ is the characteristic matrix of each layer of the proposed structure ( $\mathrm{k}=1$ to $\mathrm{n})$. A is the total TMM.

The characteristic matrix of each layer can be calculated as follows:

$$
a_{k}=\left(\begin{array}{cc}
\cos \varnothing_{k} & -\frac{i}{p_{k}} \sin \varnothing_{k} \\
-i p_{k} \sin \varnothing_{k} & \cos \varnothing_{k}
\end{array}\right),
$$

where phase difference $\left(\varnothing_{k}\right)$ is: 

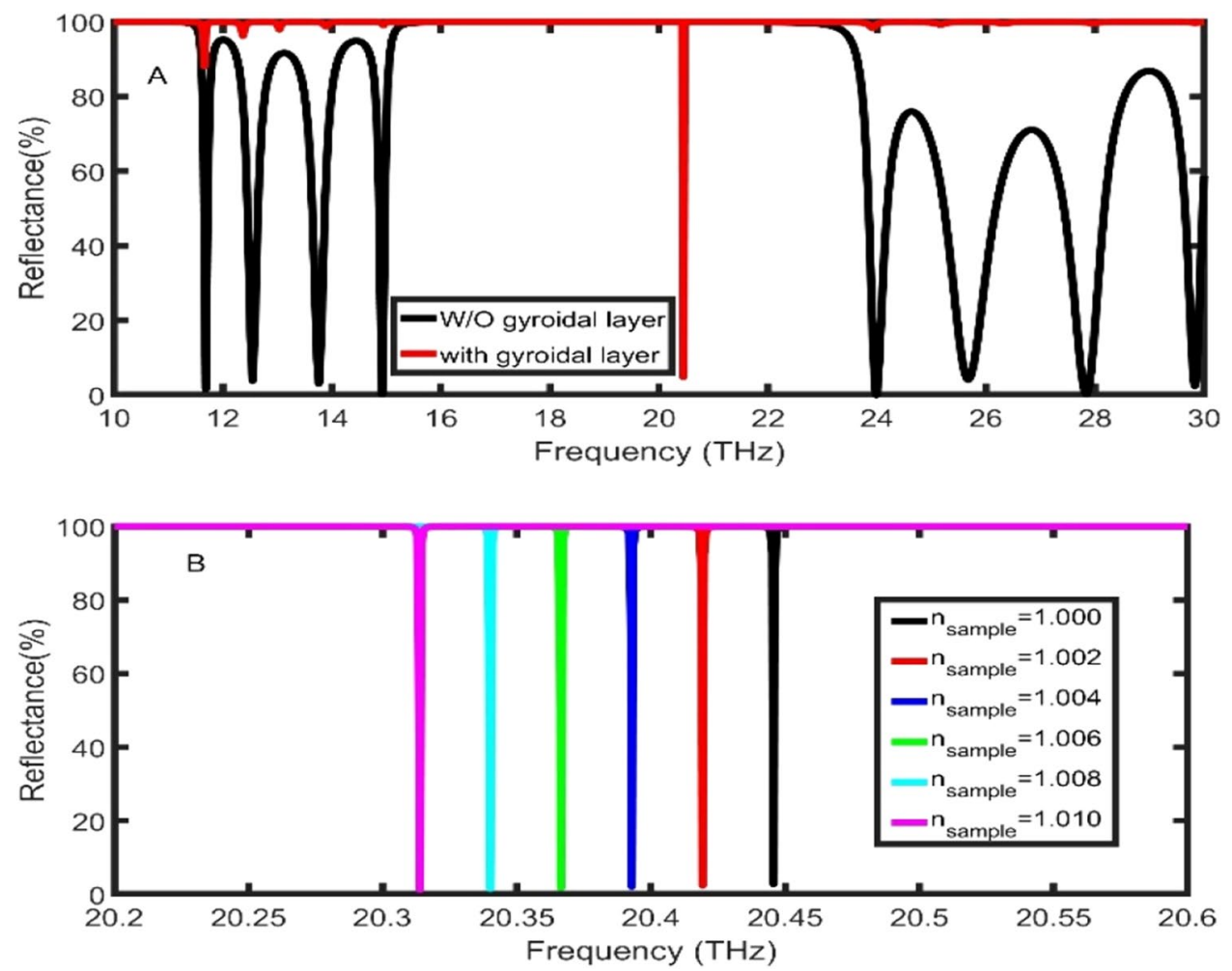

Figure 2. The reflectance spectra of the proposed PSi-PC structure (A) without GGr (black line) and with GGr (red line) at nsample $=1.00$, (B) with GGr at different values of sample layer index of refraction.

$$
\varnothing_{k}=\frac{2 \pi n_{k} d_{k} \cos \varphi_{k}}{\lambda}
$$

where $n_{k}, \varphi_{k}$ and $d_{z}$ are the index of refraction, incident angle, and thickness of each layer. The following is the light reflectance of the suggested sensor:

$$
R(\%)=100 *\lfloor r\rfloor^{2},
$$

where $r$ is the reflection coefficient.

Ethics declarations. This article does not contain any studies involving animals or human participants performed by any of the authors.

\section{Results and discussions}

The thicknesses of layers $\mathrm{GGr}_{1}$, PSi, cavity, $\mathrm{GGr}_{2}$ are $90 \mathrm{~nm}, 4500 \mathrm{~nm}, 12,000 \mathrm{~nm}$, and $400 \mathrm{~nm}$, respectively. Besides, the filling factors of $\mathrm{GGr}_{1} \mathrm{GGr}_{2}$, PSi are $3 \%, 20 \%$, and $50 \%$, respectively. The space areas inside the layers $\mathrm{GGr}_{1}, \mathrm{GGr}_{2}, \mathrm{PSi}$, and cavity layer will be filled with the gas sample ( $\left.\mathrm{n}_{\text {sample }}\right)$. The unit cell size of gyroid (a) is $30 \mathrm{~nm}, E_{F}=1 \mathrm{eV}, \tau=1 P s$, and $\mathrm{G}=1$ (monolayer of graphene).

As clear in Fig. 2A, the proposed structure without $\mathrm{GGr}_{2}$ possesses a photonic bandgap (PBG) with a bandwidth of $7.53 \mathrm{THz}$ and $100 \%$ reflectance. By adding the $\mathrm{GGr}_{2}$ layer as a plasmonic layer, the TPP dip appears inside the frequency range of the PBG at $20.45 \mathrm{THz}$ and $4.8 \%$ reflectance. Appearing of TPP dip is due to the trapping of light at the interface as a result of the PBG in the PSi-PC and strong attenuation of electromagnetic waves in graphene.

As the position of the TPP dip is strongly dependent on the effective refractive index of the whole structure, the resonant dip can be tuned with any change in the refractive index of the analyte inside the pores of the layers. As clear in Fig. 2B, by increasing the refractive index of the gas sample from 1.00 to 1.01, the TPP dip is red-shifted from 20.45 to $20.31 \mathrm{THz}$. Many parameters such as the sensitivity (S), and the figure of merit (FoM) can show the sensor performance as the following ${ }^{48,49}$ :

$$
S=\frac{\Delta f_{R}}{\Delta n_{s}}
$$



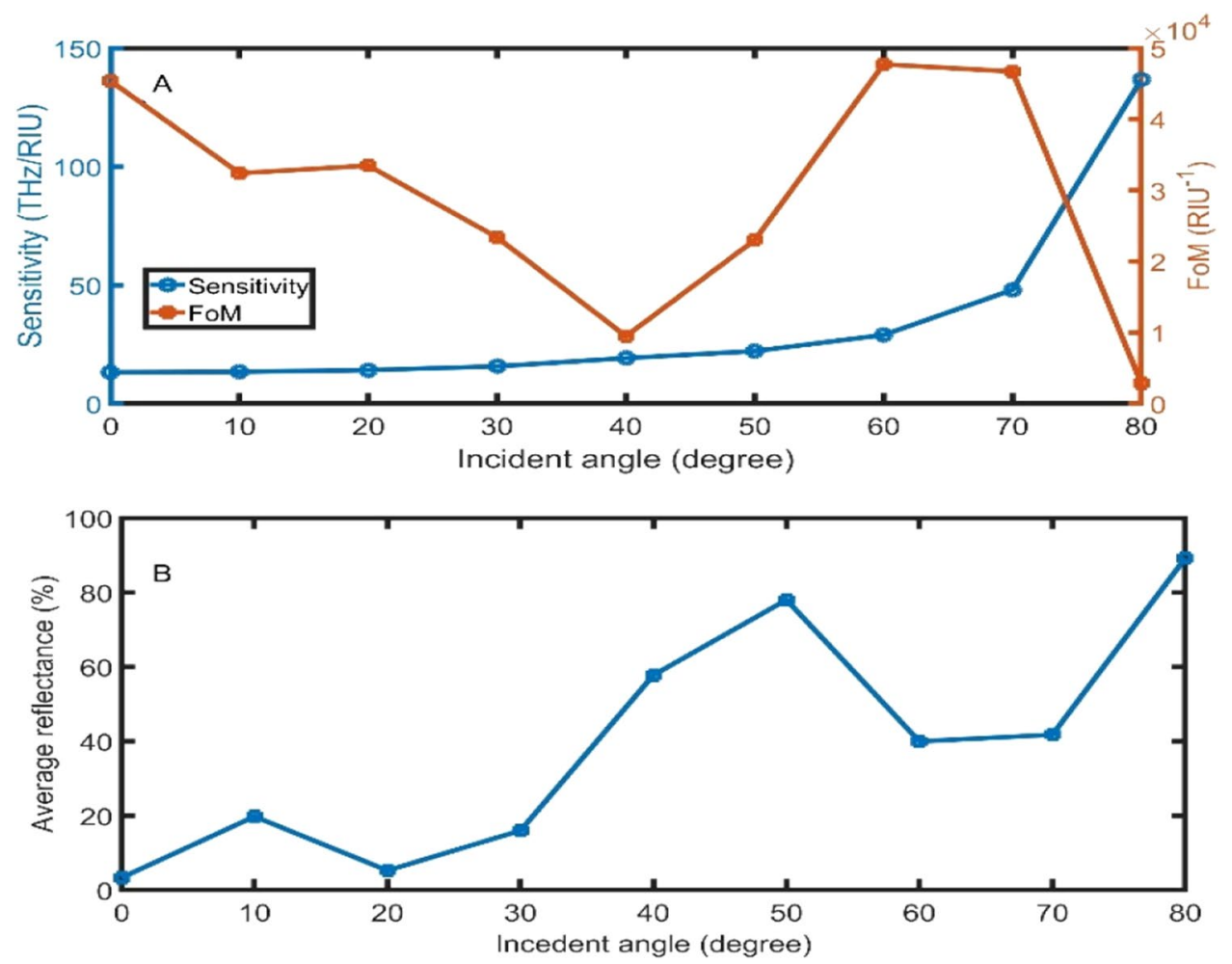

Figure 3. The (A) sensitivity and FoM, and (B) average reflectance of the proposed sensor as a function of the incident angle by changing the sample refractive index from 1.00 to 1.01 .

$$
F o M=\frac{S}{F W H M},
$$

where $\Delta f_{R}, \Delta n_{s}$, and FWHM are the average change in TPP dip position, the average change in the sample refractive index, and the bandwidth of the TPP dips, respectively. At these calculations, the proposed sensor records sensitivity of $13.19 \mathrm{THz} / \mathrm{RIU}$ and FoM of $4.5 \times 10^{4} \mathrm{RIU}^{-1}$. To enhance these values of parameters, the effect of the incident angle, the thickness of the sample layer, and the thickness of $\mathrm{GGr}_{1}$ on the performance will be numerically studied.

Changing the incident angle is an effective way to control and enhance the performance of the TPP sensors ${ }^{16,17}$. The reflectance of experimentally PC was studied at the angle of incidence range from $0^{\circ}$ to $90^{\circ}$ in many works ${ }^{50,51}$. Within the proposed structure, the optical path length of the electromagnetic wave increases when the incidence angle increases. In addition, the confinement of the electromagnetic waves within the analyte increases ${ }^{16}$. Due to the previous results, the sensitivity increases from $13.19 \mathrm{THz} / \mathrm{RIU}$ to $136.71 \mathrm{THz} / \mathrm{RIU}$ with the increase of incident angle from $0^{\circ}$ to $80^{\circ}$ as clear in Fig. 3A. For angels higher than $80^{\circ}$, the resonant dip disappears. Besides, the FoM records the highest values at angles $0^{\circ}, 60^{\circ}$, and $70^{\circ}$, and the lowest values at $40^{\circ}$ and $80^{\circ}$. Figure $3 \mathrm{~B}$ describes the average reflectance of the two resonant dips ( at $n_{\text {sample }}=1.00$ and $n_{\text {sample }}=1.01$ ). Even though the angle of $80^{\circ}$ has a high average reflectance $(89 \%)$, the angle of $80^{\circ}$ will be the optimum angle to achieve the highest sensitivity.

As clear in Fig. 4A, the sensitivity of the gyroidal graphene PSi-PC increases from $136.71 \mathrm{THz} / \mathrm{RIU}$ to 177.95 $\mathrm{THz} / \mathrm{RIU}$ with increasing the thickness of the sample layer thickness from 1200 to $16,000 \mathrm{~nm}$. But the sensitivity decreases to $139.44 \mathrm{THz} / \mathrm{RIU}$ with increasing the thickness of the sample layer to $22,000 \mathrm{~nm}$. In addition, the FoM rapidly increases from 2848 to $\mathbf{1 3 1 , 0 5 2} \mathrm{RIU}^{-1}$ with increasing the thickness from 12,000 to $20,000 \mathrm{~nm}$. Further increases in the sample thickness will have a slight effect on the FoM. For the average reflectance, it decreases from 89 to $50 \%$ with increasing the thickness of the sample layer from 12,000 nm to 20,000 nm. Then, average reflectance increases for further thickness increase, as clear in Fig. 4B. We have strongly recommended the optimum thickness of $16,000 \mathrm{~nm}$ because it recorded the highest sensitivity.

Herein, the effect of the gyroidal layer thickness $\left(\mathrm{GGr}_{1}\right)$ is investigated, as clearly visible in Fig. 5 . With the increase in the gyroidal layer thickness from 90 to $125 \mathrm{~nm}$, both for sensitivity and FoM, the performance of the proposed sensor is dramatically enhanced to record sensitivity of $188.78 \mathrm{THz} / \mathrm{RIU}$ and FoM of $355,384 \mathrm{RIU}^{-1}$, as clear in Fig. 5A. Even though the thickness of $125 \mathrm{~nm}$ does not record the highest values of sensitivity and FoM, it will be the optimum thickness of the gyroidal layer of graphene because it has the lowest reflectance, as clear in Fig. 5B.

As clear in Table 1, at different values of refractive indices, the Sensitivity and FoM will be studied for the proposed sensor. By increasing $\mathrm{n}_{\text {sample }}$ from 1.01 to 1.10 , the sensitivity and FoM decrease from $188.76 \mathrm{THz} / \mathrm{RIU}$ to $33.00 \mathrm{THz} / \mathrm{RIU}$ and from 635,984 to $7535 \mathrm{RIU}^{-1}$, respectively. As clear in Fig. 6, the highest performance was recorded at low values of $\mathrm{n}_{\text {sample }}$. Besides, with the increase of refractive index value, the reflectance of the TPP 

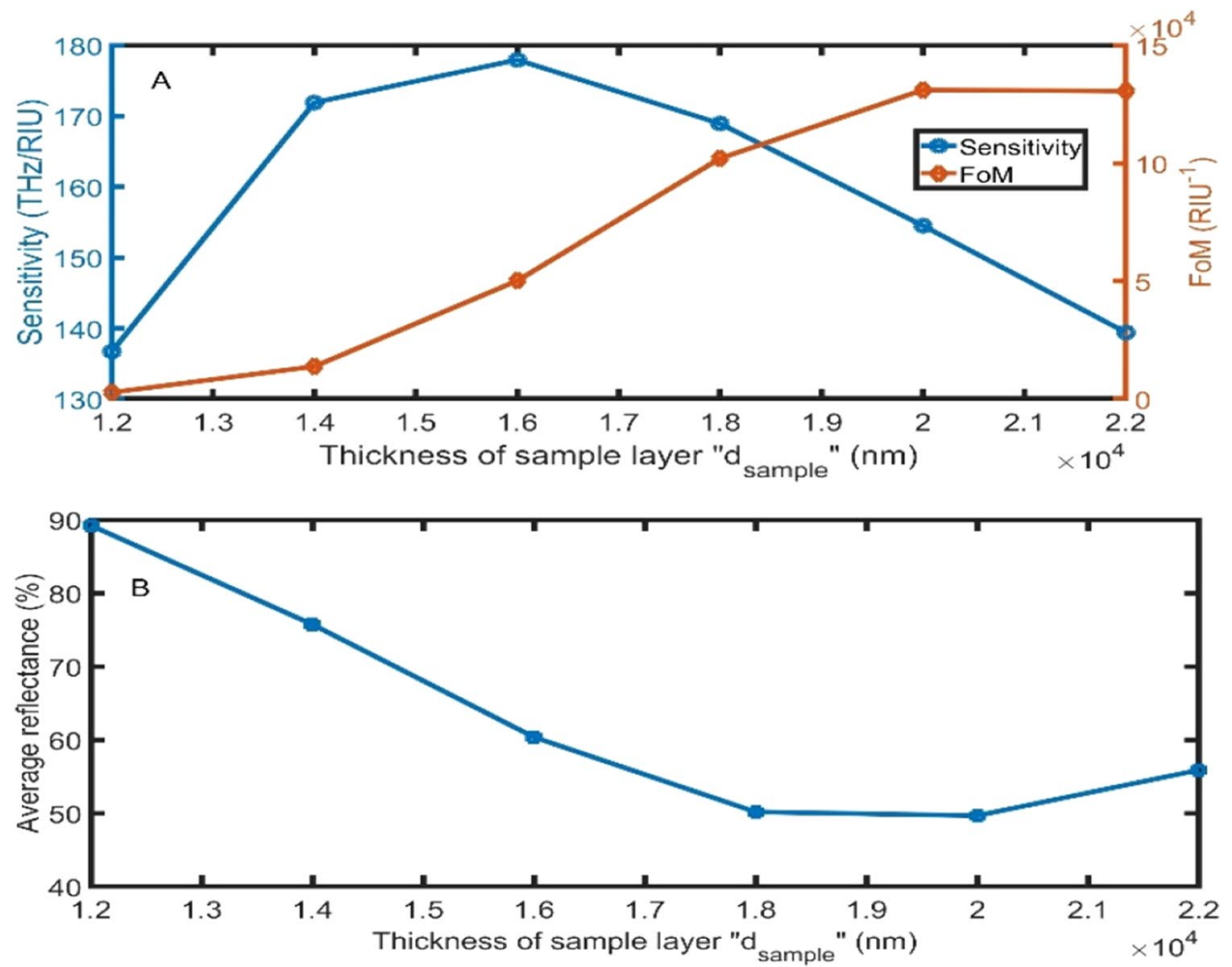

Figure 4. The (A) sensitivity and FoM, and (B) average reflectance of the proposed sensor as a function of the sample layer thickness by changing the sample refractive index from 1.00 to 1.01 at $80^{\circ}$.
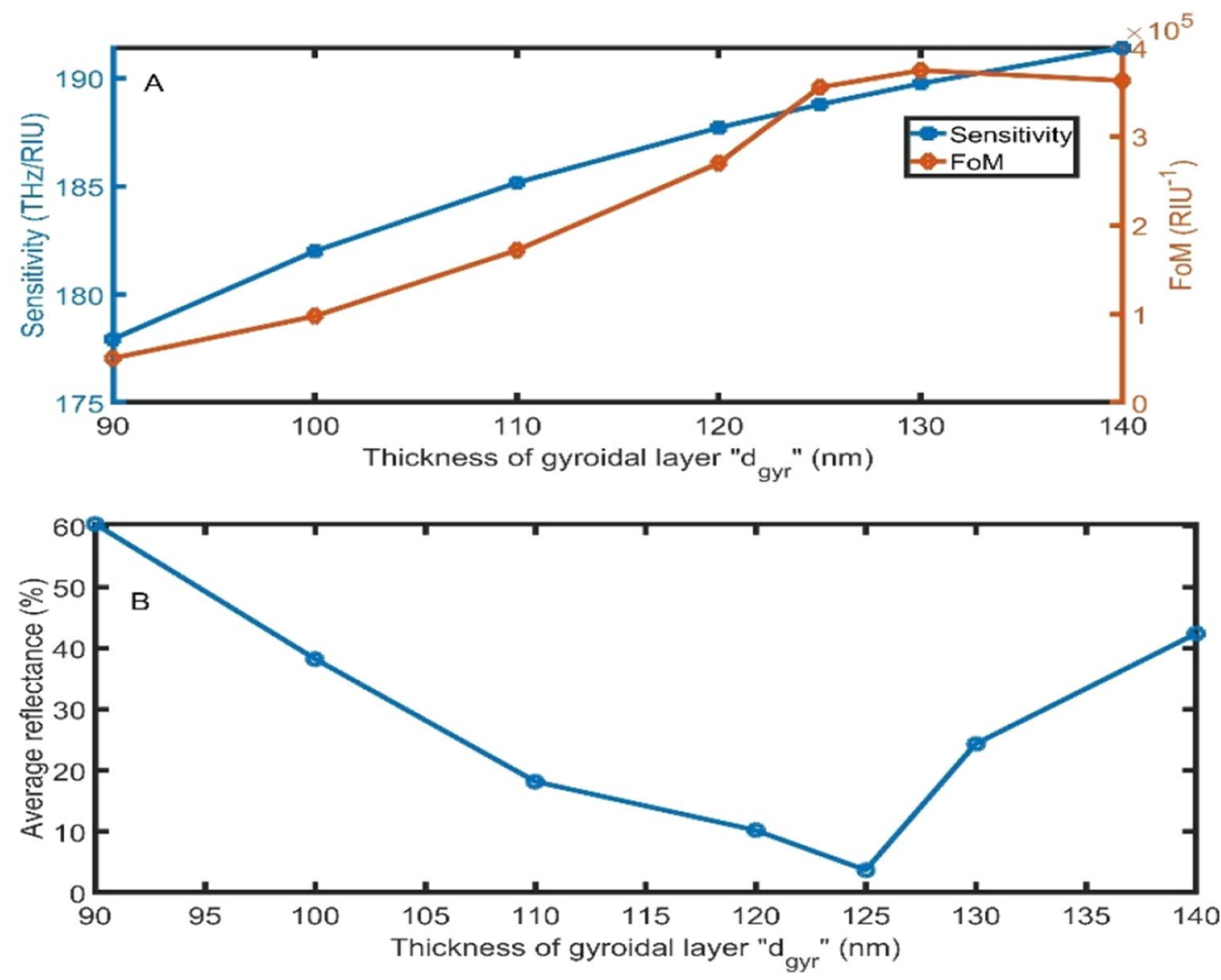

Figure 5. The (A) sensitivity and FoM, and (B) average reflectance of the proposed sensor as a function of the gyroidal graphene layer thickness by changing the sample refractive index from 1.00 to 1.01 at $80^{\circ}$ and $\mathrm{d}_{\text {sample }}=16,000 \mathrm{~nm}$. 


\begin{tabular}{|l|l|l|}
\hline $\mathbf{n}_{\text {sample }}$ & $\mathbf{S}(\mathrm{THz} / \mathrm{RIU})$ & FoM $\left(\mathbf{R I U}^{-1}\right)$ \\
\hline 1.00 & - & - \\
\hline 1.01 & 188.76 & 635,984 \\
\hline 1.02 & 107.81 & 376,616 \\
\hline 1.03 & 74.58 & 241,547 \\
\hline 1.04 & 58.69 & 163,578 \\
\hline 1.05 & 49.95 & 108,933 \\
\hline 1.06 & 44.52 & 70,880 \\
\hline 1.07 & 40.75 & 44,011 \\
\hline 1.08 & 37.79 & 25,843 \\
\hline 1.9 & 35.24 & 14,329 \\
\hline 1.10 & 33.00 & 7535 \\
\hline
\end{tabular}

Table 1. Sensitivity and FoM at different ranges of $\mathrm{n}_{\text {sample: }}$ :

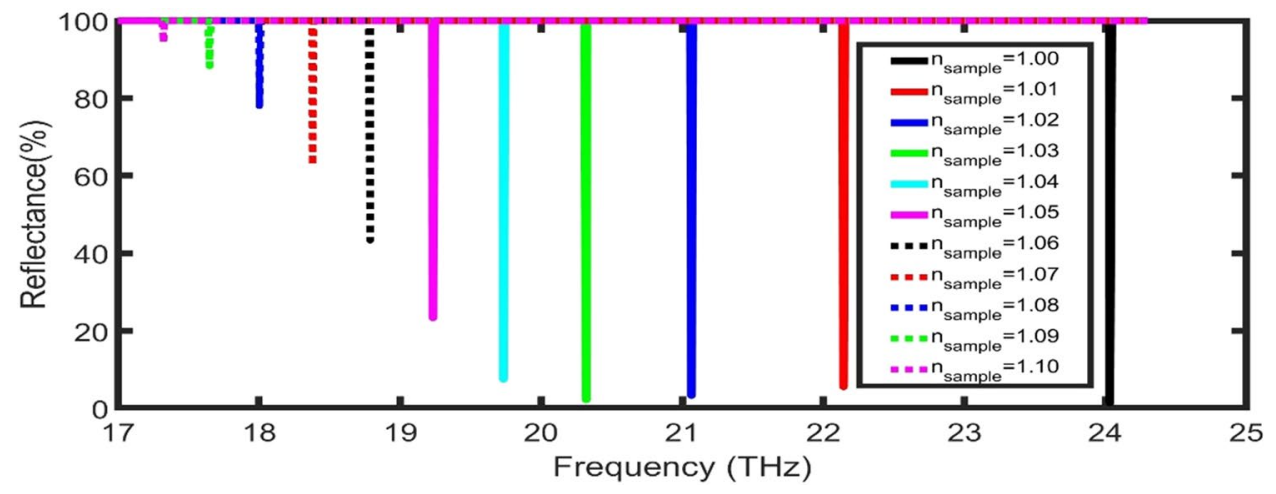

Figure 6. The reflectance spectra of the proposed sensor at different values of refractive indices.

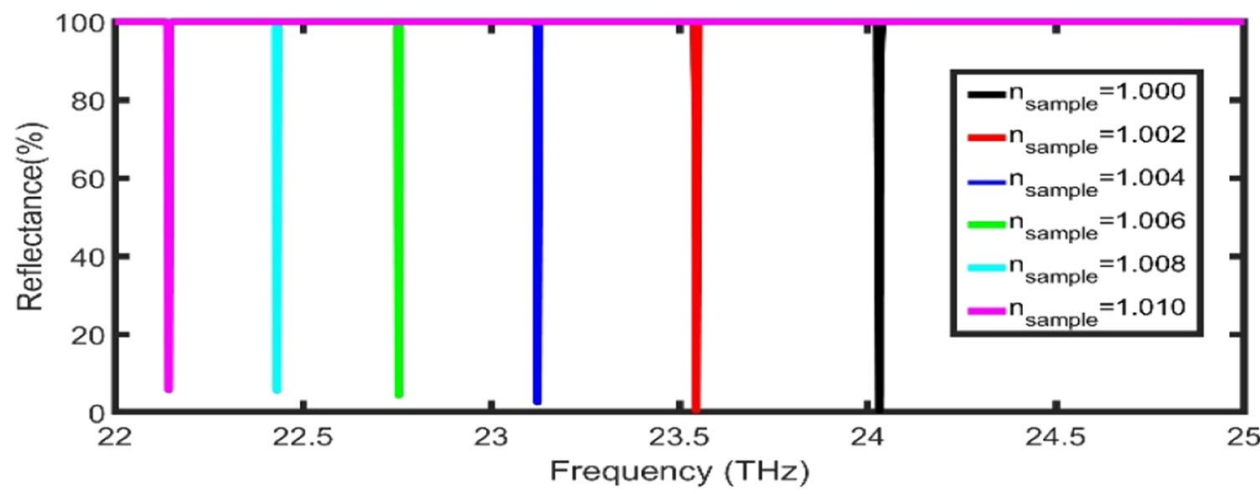

Figure 7. The reflectance spectra of the proposed sensor at the selected geometrical parameters.

dips decrease. So, the proposed structure can be used in gas sensing and air pollution applications in refractive index range from 1.00 to 1.01 .

Finally, in Fig. 7, the reflectance spectra of the proposed sensor at the selected geometrical parameters are plotted as a function of frequency. The sensitivity and FoM are much larger than many published papers, as clear in Table 2.

TPP is shifted to lower or higher frequencies with a tiny change in the effective index of refraction of the whole geometrical structure or the medium that surrounds the structure ${ }^{5}$. The effective index of refraction of the whole geometrical structure is the summation of the index of refraction of each layer multiplied by the volume fraction of this layer in the structure. So, we have tried to increase the volume fraction of the sample in the structure by using a wide sample layer. Besides, we used porous silicon and gyroidal graphene filled with sample analyte. 


\begin{tabular}{|l|l|l|l|l|}
\hline Reference & S (THz/RIU) & FoM $\left(\right.$ RIU $\left.^{-1}\right)$ & Refractive index range & Materials \\
\hline${ }^{36}, 2019$ & 0.7 & 10.3 & 1.00 to 1.10 & Tamm with graphene \\
\hline${ }^{52}, 2020$ & 0.9 & NC & 1.15 to 1.76 & Dirac semimetal \\
\hline${ }^{53}, 2020$ & 1.0 & NC & 1.15 to 1.76 & Graphene-metastructure \\
\hline${ }^{54}, 2020$ & 0.3 & 8.4 & 1.00 to 2.44 & Metamaterial \\
\hline${ }^{55}, 2020$ & 0.5 & 12.4 & 1.35 to 1.49 & Dielectric metasurface \\
\hline${ }^{56}, 2020$ & 1.6 & 24.5 & 1.00 to 1.80 & Graphene disks \\
\hline${ }^{57}, 2020$ & 1.7 & 7 & 1.00 to 2.00 & Graphene metamaterials \\
\hline${ }^{58}, 2020$ & 1.0 & 14.1 & 1.00 to 2.20 & Stacked metamaterials \\
\hline${ }^{17}, 2021$ & 4.75 & 475 & 1.00 to 1.72 & Graphene and porous silicon \\
\hline Our work & 188.8 & 355,384 & 1.00 to 1.10 & Graphene gyroid and porous silicon \\
\hline
\end{tabular}

Table 2. Comparison with other designs performance (NC not be calculated).

This design helped make any tiny change in the sample refractive index strongly affect the value of the effective refractive index of the structure and increase the TPP dip shift.

\section{Conclusion}

A novel structure of 1D-PC of porous silicon/gyroidal graphene terminated with a gyroidal graphene layer was proposed for sensing applications in the THz range. The effect of the incident angle and thickness of the gyroidal and sample layer was studied. The proposed sensor recorded sensitivity and FoM higher 38\% and $747 \%$ respectively than a similar structure composed of graphene sheets and porous silicon. Another benefit of the suggested design is that it can be used at room temperature. Besides, our sensor has ultra-high performance comparing with other published papers. This study provides a new train of investigated methods for designing nanophotonic devices based on porous silicon and gyroidal graphene layers.

\section{Data availability}

Requests for materials or code should be addressed to Z.A.Z.

Received: 9 July 2021; Accepted: 7 September 2021

Published online: 29 September 2021

\section{References}

1. Zhou, H., Yang, G., Wang, K., Long, H. \& Lu, P. Multiple optical Tamm states at a metal-dielectric mirror interface. Opt. Lett. 35, 4112-4114. https://doi.org/10.1364/OL.35.004112 (2010).

2. Shukla, M. K. \& Das, R. Tamm-plasmon polaritons in one-dimensional photonic quasi-crystals. Opt. Lett. 43, 362-365. https:// doi.org/10.1364/OL.43.000362 (2018).

3. Zaky, Z. A., Sharma, A., Alamri, S. \& Aly, A. H. Theoretical evaluation of the refractive index sensing capability using the coupling of Tamm-Fano resonance in one-dimensional photonic crystals. Appl. Nanosci. 11, 2261-2270. https://doi.org/10.1007/s13204021-01965-7 (2021).

4. Zaky, Z. A. \& Aly, A. H. Highly sensitive salinity and temperature sensor using Tamm resonance. Plasmonics https://doi.org/10. 1007/s11468-021-01487-6 (2021).

5. Zaky, Z. A., Ahmed, A. M. \& Aly, A. H. Remote temperature sensor based on Tamm resonance. SILICON https://doi.org/10.1007/ s12633-021-01064-w (2021).

6. Zaky, Z. A. \& Aly, A. H. Theoretical study of a tunable low-temperature photonic crystal sensor using dielectric-superconductor nanocomposite layers. J. Supercond. Nov. Magn 33, 2983-2990. https://doi.org/10.1007/s10948-020-05584-1 (2020).

7. Zhang, X.-L., Song, J.-F., Li, X.-B., Feng, J. \& Sun, H.-B. Optical Tamm states enhanced broad-band absorption of organic solar cells. Appl. Phys. Lett. 101, 243901. https://doi.org/10.1063/1.4770316 (2012).

8. Symonds, C. et al. Confined Tamm plasmon lasers. Nano Lett. 13, 3179-3184. https://doi.org/10.1021/nl401210b (2013).

9. Zhang, X.-L. et al. Hybrid Tamm plasmon-polariton/microcavity modes for white top-emitting organic light-emitting devices. Optica 2, 579-584. https://doi.org/10.1364/OPTICA.2.000579 (2015).

10. Juneau-Fecteau, A., Savin, R., Boucherif, A. \& Fréchette, L. G. A practical Tamm plasmon sensor based on porous Si. AIP Adv. 11, 065305. https://doi.org/10.1063/5.0054629 (2021).

11. Aly, A. H., Zaky, Z. A., Shalaby, A. S., Ahmed, A. M. \& Vigneswaran, D. Theoretical study of hybrid multifunctional one-dimensional photonic crystal as a flexible blood sugar sensor. Phys. Scr. 95, 035510. https://doi.org/10.1088/1402-4896/ab53f5 (2020).

12. Aly, A. H. \& Zaky, Z. A. Ultra-sensitive photonic crystal cancer cells sensor with a high-quality factor. Cryogenics 104, 102991. https://doi.org/10.1016/j.cryogenics.2019.102991 (2019).

13. Aly, A. H. et al. Novel biosensor detection of tuberculosis based on photonic band gap materials. Mater. Res.-Ibero-Am. J. Mater. 24, e20200483. https://doi.org/10.1590/1980-5373-MR-2020-0483 (2021).

14. Abd El-Ghany, S. E., Noum, W. M., Matar, Z., Zaky, Z. A. \& Aly, A. H. Optimized bio-photonic sensor using 1D-photonic crystals as a blood hemoglobin sensor. Phys. Scr. 96, 035501. https://doi.org/10.1088/1402-4896/abd49c (2020).

15. Tammam, M. T. et al. in IOP Conference Series: Materials Science and Engineering. Vol. 1171. 012005. https://doi.org/10.1088/ 1757-899X/1171/1/012005 (2021).

16. Zaky, Z. A., Ahmed, A. M., Shalaby, A. S. \& Aly, A. H. Refractive index gas sensor based on the Tamm state in a one-dimensional photonic crystal: Theoretical optimisation. Sci. Rep. 10, 9736. https://doi.org/10.1038/s41598-020-66427-6 (2020).

17. Zaky, Z. A. \& Aly, A. H. Modeling of a biosensor using Tamm resonance excited by graphene. Appl. Opt. 60, 1411-1419. https:// doi.org/10.1364/AO.412896 (2021).

18. Jenie, S. A. et al. Lanthanide luminescence enhancements in porous silicon resonant microcavities. ACS Appl. Mater. Interfaces 6, 12012-12021. https://doi.org/10.1021/am500983r (2014). 
19. Verma, C. P. et al. Cavity resonance tunability of porous silicon microcavities by Ar+ ion irradiation. Appl. Surf. Sci. https://doi. org/10.1016/j.apsusc.2020.147696 (2020).

20. Bikbaev, R. G., Vetrov, S. Y. \& Timofeev, I. V. Optical Tamm states at the interface between a photonic crystal and a gyroid layer. J. Opt. Soc. Am. B-Opt. Phys. 34, 2198-2202. https://doi.org/10.1364/JOSAB.34.002198 (2017).

21. Sun, P. et al. 3D interconnected gyroid Au-CuS materials for efficient solar steam generation. ACS Appl. Mater. Interfaces 12, 34837-34847. https://doi.org/10.1021/acsami.0c06701 (2020).

22. Dolan, J. A. et al. Optical properties of gyroid structured materials: from photonic crystals to metamaterials. Adv. Opt. Mater. 3, 12-32. https://doi.org/10.1002/adom.201400333 (2015).

23. Turner, M. D. et al. Miniature chiral beamsplitter based on gyroid photonic crystals. Nat. Photon. 7, 801-805. https://doi.org/10. 1038/nphoton.2013.233 (2013).

24. Cebo, T. et al. Chemical vapour deposition of freestanding sub-60 nm graphene gyroids. Appl. Phys. Lett. 111, 253103. https://doi. org/10.1063/1.4997774 (2017).

25. Nakanishi, K. et al. Mechanical properties of the hollow-wall graphene gyroid lattice. Acta Mater. 201, 254-265. https://doi.org/ 10.1016/j.actamat.2020.09.077 (2020).

26. Ma, S. et al. Mechanical behaviours and mass transport properties of bone-mimicking scaffolds consisted of gyroid structures manufactured using selective laser melting. J. Mech. Behav. Biomed. Mater. 93, 158-169. https://doi.org/10.1016/j.jmbbm.2019. 01.023 (2019).

27. Feng, Z. et al. Fabrication of graphene-reinforced nanocomposites with improved fracture toughness in net shape for complex 3D structures via digital light processing. C.-J. Carbon Res. 5, 25. https://doi.org/10.3390/c5020025 (2019).

28. Hensleigh, R. M. et al. Additive manufacturing of complex micro-architected graphene aerogels. Mater. Horiz. 5, 1035-1041. https://doi.org/10.1039/C8MH00668G (2018).

29. Liu, K., Chen, Y.-M., Policastro, G. M., Becker, M. L. \& Zhu, Y. Three-dimensional bicontinuous graphene monolith from polymer templates. ACS Nano 9, 6041-6049. https://doi.org/10.1021/acsnano.5b01006 (2015).

30. Zhang, H. et al. Z-scan measurement of the nonlinear refractive index of graphene. Opt. Lett. 37, 1856-1858. https://doi.org/10. 1364/OL.37.001856 (2012).

31. Li, Z. et al. Dirac charge dynamics in graphene by infrared spectroscopy. Nat. Phys. 4, 532-535. https://doi.org/10.1038/nphys989 (2008).

32. Lu, G., Yu, K., Wen, Z. \& Chen, J. Semiconducting graphene: Converting graphene from semimetal to semiconductor. Nanoscale 5, 1353-1368. https://doi.org/10.1039/C2NR32453A (2013).

33. Jiang, L. et al. Manipulating optical Tamm state in the terahertz frequency range with graphene. Chin. Opt. Lett. 17, 020008. https:// doi.org/10.1364/COL.17.020008 (2019).

34. Bao, Q. \& Loh, K. P. Graphene photonics, plasmonics, and broadband optoelectronic devices. ACS Nano 6, 3677-3694. https:// doi.org/10.1021/nn300989g (2012).

35. Keshavarz, M. M. \& Alighanbari, A. Self-referenced terahertz refractive index sensor based on a cavity resonance and Tamm plasmonic modes. Appl. Opt. 59, 4517-4526. https://doi.org/10.1364/AO.391210 (2020).

36. Keshavarz, M. M. \& Alighanbari, A. Terahertz refractive index sensor based on Tamm plasmon-polaritons with graphene. Appl. Opt. 58, 3604-3612. https://doi.org/10.1364/AO.58.003604 (2019).

37. Abueidda, D. W. et al. Mechanical properties of 3D printed polymeric gyroid cellular structures: Experimental and finite element study. Mater. Des. 165, 107597 (2019).

38. Farah, P. et al. Ultrafast nonlinear response of gold gyroid three-dimensional metamaterials. Phys. Rev. Appl. 2, 044002. https:// doi.org/10.1103/PhysRevApplied.2.044002 (2014).

39. Bludov, Y. V., Ferreira, A., Peres, N. M. \& Vasilevskiy, M. I. A primer on surface plasmon-polaritons in graphene. Int. J. Mod. Phys. B 27, 1341001. https://doi.org/10.1142/S0217979213410014 (2013).

40. Ye, Y., Xie, M., Tang, J. \& Ouyang, J. Highly sensitive and tunable terahertz biosensor based on optical Tamm states in graphenebased Bragg reflector. Results Phys. 15, 102779. https://doi.org/10.1016/j.rinp.2019.102779 (2019).

41. Ahmed, A. M. \& Mehaney, A. Ultra-high sensitive 1D porous silicon photonic crystal sensor based on the coupling of Tamm/Fano resonances in the mid-infrared region. Sci. Rep. 9, 6973. https://doi.org/10.1038/s41598-019-43440-y (2019).

42. Salem, M., Sailor, M., Harraz, F., Sakka, T. \& Ogata, Y. Electrochemical stabilization of porous silicon multilayers for sensing various chemical compounds. J. Appl. Phys. 100, 083520. https://doi.org/10.1063/1.2360389 (2006).

43. Li, J., He, J. \& Hong, Z. Terahertz wave switch based on silicon photonic crystals. Appl. Opt. 46, 5034-5037. https://doi.org/10. 1364/AO.46.005034 (2007).

44. Aly, A. H., Ryu, S.-W. \& Wu, C.-J. Electromagnetic wave propagation characteristics in a one-dimensional metallic photonic crystal. J. Nonlinear Opt. Phys. Mater. 17, 255-264. https://doi.org/10.1142/S0218863508004160 (2008).

45. Yeh, P. Optical Waves in Layered Media Vol. 95 (Wiley, 1988).

46. Born, M. \& Wolf, E. Principles of Optics: Electromagnetic Theory of Propagation, Interference and Diffraction of Light (Elsevier, 2013).

47. Yeh, P., Yariv, A. \& Hong, C.-S. Electromagnetic propagation in periodic stratified media. I. General theory. J. Opt. Soc. Am. B-Opt. Phys. 67, 423-438. https://doi.org/10.1364/JOSA.67.000423 (1977).

48. Ayyanar, N., Raja, G. T., Sharma, M. \& Kumar, D. S. Photonic crystal fiber-based refractive index sensor for early detection of cancer. IEEE Sens. J. 18, 7093-7099. https://doi.org/10.1109/JSEN.2018.2854375 (2018).

49. Panda, A. \& Devi, P. P. Photonic crystal biosensor for refractive index based cancerous cell detection. Opt. Fiber Technol. 54, 102123. https://doi.org/10.1016/j.yofte.2019.102123 (2020).

50. Kim, Y. et al. Air-like plasmonics with ultralow-refractive-index silica aerogels. Sci. Rep. 9, 1-9 (2019).

51. Byun, K., Kim, N.-H., Leem, J. \& Yu, J. Enhanced surface plasmon resonance detection using porous ITO-gold hybrid substrates. Appl. Phys. B-Lasers Opt. 107, 803-808. https://doi.org/10.1007/s00340-012-4998-5 (2012).

52. Yan, S., Song, S., Zhang, H. \& Zhang, Y. Dynamically tunable Fano resonance with high Q factor based on asymmetric Dirac semimetal split-ring structure. Mater. Res. Express 7, 025041. https://doi.org/10.1088/2053-1591/ab74fe (2020).

53. Asgari, S., Granpayeh, N. \& Fabritius, T. Controllable terahertz cross-shaped three-dimensional graphene intrinsically chiral metastructure and its biosensing application. Opt. Commun. https://doi.org/10.1016/j.optcom.2020.126080 (2020).

54. Chen, T., Zhang, D., Huang, F., Li, Z. \& Hu, F. Design of a terahertz metamaterial sensor based on split ring resonator nested square ring resonator. Mater. Res. Express 7, 095802. https://doi.org/10.1088/2053-1591/abb496 (2020).

55. Wang, Y. et al. Properties and sensing performance of all-dielectric metasurface THz absorbers. IEEE Trans. Terahertz Sci. Technol. 10, 599-605. https://doi.org/10.1109/TTHZ.2020.3010164 (2020).

56. Rezagholizadeh, E., Biabanifard, M. \& Borzooei, S. Analytical design of tunable THz refractive index sensor for TE and TM modes using graphene disks. J. Phys. D-Appl. Phys. 53, 295107. https://doi.org/10.1088/1361-6463/ab85e6 (2020).

57. He, Z. et al. Ultra-high sensitivity sensing based on tunable plasmon-induced transparency in graphene metamaterials in terahertz. Opt. Mater. 108, 110221. https://doi.org/10.1016/j.optmat.2020.110221 (2020).

58. Karmakar, S., Kumar, D., Varshney, R. \& Chowdhury, D. R. Strong terahertz matter interaction induced ultrasensitive sensing in fano cavity based stacked metamaterials. J. Phys. D-Appl. Phys. 53, 415101. https://doi.org/10.1088/1361-6463/ab94e3 (2020). 


\section{Acknowledgements}

The authors thank the reviewers and editors for improving this article.

\section{Author contributions}

Z.A.Z. corresponding author, invented the original idea of the study, implemented the computer code, performed the numerical simulations, analyzed the data, wrote and revised the main manuscript text. A.H.A. reviewing, discussed the results, and supervised this work. All authors developed the final manuscript.

\section{Competing interests}

The authors declare no competing interests.

\section{Additional information}

Correspondence and requests for materials should be addressed to Z.A.Z.

Reprints and permissions information is available at www.nature.com/reprints.

Publisher's note Springer Nature remains neutral with regard to jurisdictional claims in published maps and institutional affiliations.

(c) (1) Open Access This article is licensed under a Creative Commons Attribution 4.0 International License, which permits use, sharing, adaptation, distribution and reproduction in any medium or format, as long as you give appropriate credit to the original author(s) and the source, provide a link to the Creative Commons licence, and indicate if changes were made. The images or other third party material in this article are included in the article's Creative Commons licence, unless indicated otherwise in a credit line to the material. If material is not included in the article's Creative Commons licence and your intended use is not permitted by statutory regulation or exceeds the permitted use, you will need to obtain permission directly from the copyright holder. To view a copy of this licence, visit http://creativecommons.org/licenses/by/4.0/.

(C) The Author(s) 2021 